\title{
Fatores desencadeantes de estresse do enfermeiro na unidade de urgência e emergência
}

\section{Stressful factors for nurses in the emergency and emergency unit}

\author{
Tatiana Aparecida Freitas Garçon' $\bullet$ Luiz Alegria Aguiar ${ }^{2} \bullet$ Eliseu Santos do Nascimento $^{3} \bullet$ Aline Voltarelli $^{4}$
}

\begin{abstract}
RESUMO
A unidade de Urgência e Emergência é uma unidade na qual chegam clientes com diversas doenças, e com necessidades diversificadas, sendo elas emergenciais ou não, cabendo aos profissionais que atuam dentro da unidade saber identificar e atender os pacientes mediante suas necessidades. Trata-se de uma revisão integrativa, com recorte temporal entre 2011 a 2016, acessíveis eletronicamente. Os conflitos de funções levam à insatisfação no trabalho e relações inter pessoais, a desvalorização profissional pode levar à desmotivação e abandono do trabalho, ocasionando altas taxas de absenteísmo, também ao ambiente insalubre, o que gera, na maioria das vezes, sobrecarga de trabalho para uns e priorizando outros. O Profissional Enfermeiro no setor de emergência percebe os fatores de estresse nos quais está envolvido, e é de grande valia o estímulo como solução para melhor qualidade de vida do profissional de enfermagem, quando a instituição na qual está o trabalhador vinculado dispor de plano de carreira, aumento salarial adequado para seu nível de estudo, liderança democrática e não punitiva, assim como um ambiente sustentável contribuindo para que este profissional tenha qualidade de vida.
\end{abstract}

Palavras-chave: Enfermeiro; Emergência; Estresse Profissional.

\begin{abstract}
The Emergency and Emergency unit is a unit in which clients with different diseases arrive and with diverse needs, whether they are emergency or not, it is up to the professionals who work within the unit to know how to identify and attend to patients according to their needs. It is an integrative review, with a temporal cut between 20II and 20I6, accessible electronically. Conflicts of functions lead to job dissatisfaction and interpersonal relationships, professional devaluation can lead to demotivation and abandonment of work, causing high rates of absenteeism, also to the unhealthy environment, which generates, in most cases, work overload for some and prioritizing others. The Nursing Professional in the emergency sector perceives the stress factors in which they are involved, and it is of great value the stimulus as a solution for a better quality of life of the nursing professional, when the institution in which the worker is linked has a career plan, adequate salary for their level of study, democratic and non-punitive leadership, as well as a sustainable environment, contributing to the professional quality of life.
\end{abstract}

Keywords: Nurse; Emergency; Professional stress.

\footnotetext{
'Graduanda do Curso de Enfermagem pela Universidade Brasil Polo Fama de Mauá.Avenida Presidente Castelo Branco, 440 Jardim Zaíra - Mauá -SP Cep 0932 I-375 mail:freitastatiana@ig.com.br

${ }^{2}$ Graduando do Curso de Enfermagem pela Universdade Brasil Polo Fama de Mauá. Endereço Estrada do Mizukamy II 90 , Estância Americana, Suzano, SP, Brasil. E-mail: luizinhoct24@hotmail.com.

${ }^{3}$ Graduando do Curso de Enfermagem pela Universdade Brasil Polo Fama de Mauá. Endereço Rua. Rosa Gabioneta 439, Jd.Alto da Boa Vista, Mauá, SP, Brasil. E-mail: eliseu.snascimento@hotmail.com.

${ }^{4}$ Enfermeira. Mestre em Ciências da Saúde. Especialista em Enfermagem do Trabalho, Docência em Enfermagem Nível Técnico e Superior e Metodologia de Ensino à Distância. Docente dos Cursos de Graduação de Enfermagem e Educação Física na Faculdade FAMA/UNIESP de Mauá. Endereço Rua Michael Bispo de Oliveira número 247 Bairro Votupoca Barueri CEP 06443-007_orienta
} 


\section{INTRODUÇÃO}

A unidade de Urgência e Emergência é uma unidade na qual chegam clientes com diversas doenças, e com necessidades diversificadas, sendo elas emergenciais ou não, cabendo aos profissionais que atuam dentro da unidade saber identificar e atender os pacientes mediante suas necessidades.

É um local de atendimento em que o profissional precisafazer intervenções de urgência,desta forma, pode-se observar grande estresse nesta unidade de trabalho, nas quais as pessoas buscam atendimentoimediato. Sendo assim este ambiente torna-se estressante, pois o Enfermeiro terá de identificar quais são as necessidades primordiais e emergenciais ${ }^{(1)}$.

$\mathrm{Na}$ atualidade existem muitos serviços de saúde que funcionam durante 24 horas, e isto mostra a existência de horários de trabalho variados e trabalho noturno. Tais turnos podem ser fixos ou rotativos, em regime de turnos ou com escalas de plantão(2).

Devido a estes trabalhos em turnos, os profissionais de enfermagem podem apresentar alterações de sono, distúrbios gastrointestinais, cardiovasculares e psíquicos. Em relação à vida social e familiar destes profissionais, observa-se um prejuízo na participação de atividades sociais, faltas escolares, não participação em eventos culturais ou esportivos, o que com o tempo vai afastando ou dificultando a integração deste profissional à vida social da sua comunidade ${ }^{(3)}$.

A maioria dos prestadores de serviço da área da saúde tem dois vínculos empregatícios formalizados, por causa da diminuição no reconhecimento da sociedade e também por não terem o salário desejado, o que os sujeita às horas de trabalho extenuantes e à necessidade de adaptação com as tarefas caseiras ou até com os cursos ${ }^{(4)}$.

A Organização de Trabalho (OIT) reconhece que praticamente todas as profissões podem desenvolver estresse ocupacional, mas que a enfermagem é apontada como a profissão mais estressante.

No Brasil, segundo o decreto 3.048 de 6 de maio de 1999, que fala sobre agentes patogênicos causadores de doenças ocupacionais, a Síndrome de Burnout está classificada junto aos Transtornos Mentais e do Comportamento Relacionados com o Trabalho, manifestando-se com a sensação de exaustão. Neste caso, a Síndrome de Burnout aparece como sinônimo de Síndrome de Esgotamento Profissional, que dentre os sinais e sintomas, 0 profissional demonstra a despersonalização do estado psíquico, no qual prevalece a dissimulação afetiva ou o cinismo e uma tendência a criticar tudo e todos com quem se relaciona no trabalho(5).

Considerando as diversas funções do Enfermeiro, resultando na sobrecarga de trabalho, pode ocorrer o absenteísmo. Isso se dá ao fato de que este profissional na emergência tem diversas funções, que permeiam a coordenação clínica e funcional do setor, aplicaçãoda educação continuada junto à equipe de enfermagem, articulaçãodos diversos profissionais envolvidos na assistência a um cliente. $O$ enfermeiro também tem como função privativa a realização da sistematização de Enfermagem (SAE), ainda precisa manter atualizado o aperfeiçoamento técnico-científico, reconhecendo a responsabilidade a qual é imprescindível para o desenvolvimento da SAE, e qualidade na prestação de serviços de enfermagem ${ }^{(6)}$.

Como justificativa deste trabalho, considera-se importante identificar o real conhecimento do Enfermeiro para atuar na unidade emergência no ambiente hospitalar, bem como lidar com os fatores que desencadeiam o estresse, e como o estresse interfere nas atividades do profissional de enfermagem prestadas ao cliente.

Os objetivossão descrever os fatores de estresse do Enfermeiro no setor de emergência e identificar a influência das ações do Enfermeiro como líder na atuação dos processos do cuidado no setor de emergência.

\section{MÉTODO}

Trata-se de uma revisão integrativa, com recorte temporal entre 2011 a 2016, acessíveis eletronicamente.A revisão integrativa é um método de análise de pesquisas que possibilita a síntese do conhecimento em um determinado assunto. Inclui estudos com diferentes abordagens metodológicas, além de identificar lacunas do conhecimento que necessitam ser preenchidas com a realização de novas pesquisas ${ }^{(7)}$.

A busca foi realizada no banco de dados da SCIELO eos termos utilizados, de acordo com os descritores em ciências da saúde (DECS), são: "Enfermeiro", "Emergência", "Estresse Profissional". Todos os estudos obtidos a partir dos descritores definidos na estratégia de busca foram avaliados pelos títulos e resumos. Após a leitura dos resumos, foram selecionados os estudos seguindo os critérios de inclusão:artigosdisponíveis em português, disponíveis na integra online, e que estejam de acordo com o objetivo da pesquisa. Como critérios de exclusão: documentos que não sejam artigos, artigos duplicados ou que não estejam de acordo com o objetivo.

\section{RESULTADOS}

A busca na base de dados supracitada resultou em 18 artigos que eram pertinentes aos objetivos deste estudo, expostos na tabela I.

\section{DISCUSSÃO}

A crise do sistema de saúde no Brasil está presente no dia a dia.Pode ser constatada através de fatos amplamente conhecidos e divulgados pela mídia, como exten- 


\section{TABELA 1 - Distribuição das publicações sobre os Fatores Desencadeantes de Estresse do Enfermeiro na unidade de urgência e Emergência. Brasil, 2017.}

\begin{tabular}{|c|c|c|c|}
\hline Título & Autor (es) & Principais Resultados & Ano \\
\hline $\begin{array}{l}\text { Humanização da Saúde em um Serviço de } \\
\text { Emergência um hospital de emergência de um } \\
\text { hospital Público: Comparação sobre representações } \\
\text { sociais dos profissionais antes e após a } \\
\text { capacitação(8). }\end{array}$ & $\begin{array}{c}\text { Andrade MAC; } \\
\text { Artmann E; Trindade, } \\
\text { ZA. }\end{array}$ & $\begin{array}{l}\text { Comunicação efetiva na Gestão com } \\
\text { humanização para o trabalho que promova } \\
\text { a cultura de humanização como reforço no } \\
\text { trabalho dos profissionais de saúde igualmente } \\
\text { entre si. }\end{array}$ & 2011 \\
\hline $\begin{array}{l}\text { Estresse ocupacional dos enfermeiros de urgência e } \\
\text { emergência }{ }^{(9)} \text {. }\end{array}$ & $\begin{array}{l}\text { Bezerra, FN.; Silva, T } \\
\text { Marques; Ramos VP }\end{array}$ & $\begin{array}{l}\text { Está relacionado à escassez de } \\
\text { recursos humanos e a carga horária de } \\
\text { trabalho,instalações físicas e recursos de } \\
\text { materiais inadequados. }\end{array}$ & 2012 \\
\hline $\begin{array}{l}\text { Humanizado em Terapia Intensiva: Uma Revisão } \\
\text { Bibliográfica }^{(10)} \text {. }\end{array}$ & $\begin{array}{l}\text { Camponogara S, } \\
\text { Santos TM, Seiffert } \\
\text { MA et al. }\end{array}$ & $\begin{array}{l}\text { Revisão sobre o cuidado humanizado em terapia } \\
\text { intensiva. }\end{array}$ & 2011 \\
\hline $\begin{array}{l}\text { Estresse precoce no desenvolvimento: impactos na } \\
\text { saúde e mecanismos de proteção(11). }\end{array}$ & Linhares M & $\begin{array}{l}\text { Intervenções preventivas para manejo de } \\
\text { estresse e dor. }\end{array}$ & 2016 \\
\hline $\begin{array}{l}\text { Considerações sobre o conceito de Dignidade } \\
\text { Humana }^{(12)} \text {. }\end{array}$ & Frias L; Lopes N & $\begin{array}{l}\text { Autonomia pessoal e das condições para } \\
\text { desenvolve-las e exerce-las. }\end{array}$ & 2015 \\
\hline $\begin{array}{l}\text { Perfil de usuários atendidos em uma unidade não } \\
\text { hospitalar de urgência(2). }\end{array}$ & Garcia, VM; Reis, RK. & $\begin{array}{l}\text { Na atualidade existem muitos serviços de saúde } \\
\text { que funcionam durante } 24 \mathrm{~h} \text { horas e isto mostra } \\
\text { a existência de horários de trabalho variados e } \\
\text { trabalho noturno. Tais turnos de trabalho podem } \\
\text { ser fixos ou rotativos, em regime de turnos ou com } \\
\text { escalas de plantão. }\end{array}$ & 2014 \\
\hline $\begin{array}{l}\text { Trabalho Significativo e Felicidade Humana: } \\
\text { explorando aproximações }{ }^{(4)} \text {. }\end{array}$ & Silva N, Tolfo SR & $\begin{array}{l}\text { Bem estar e qualidade de vida no trabalho. } \\
\text { Realização humana por meio de tarefas } \\
\text { constituídasde importância para quem as realiza. }\end{array}$ & 2012 \\
\hline $\begin{array}{l}\text { Qualidade de vida no trabalho (QVT) do } \\
\text { assistencialismo à promoção efetiva La boreal(13). }\end{array}$ & Cesar FMC & $\begin{array}{l}\text { Perguntar aos trabalhadores como eles avaliam a } \\
\text { qualidade de vida no trabalho. }\end{array}$ & 2015 \\
\hline $\begin{array}{l}\text { Discussão sobre as causas da Síndrome de Burnout } \\
\text { e suas implicações à saúde de profissional de } \\
\text { Enfermagem }^{(5)} \text {. }\end{array}$ & $\begin{array}{l}\text { Lima SJL; Campos } \\
\text { DAR. }\end{array}$ & $\begin{array}{l}\text { Principais causas da Síndrome de Burnout e } \\
\text { suas implicações à saúde aos profissionais de } \\
\text { enfermagem. }\end{array}$ & 2012 \\
\hline $\begin{array}{l}\text { Indicadores de absenteísmo e diagnósticos } \\
\text { associados às licenças médicas de trabalhadores da } \\
\text { área de serviços de uma indústria de petróleo. }{ }^{(14)} \text {. }\end{array}$ & $\begin{array}{l}\text { Oenning, NS; } \\
\text { Carvalho FM; Lima } \\
\text { VMC. }\end{array}$ & $\begin{array}{l}\text { A importância das doenças ocupacionais no } \\
\text { absenteísmo por licença médica. }\end{array}$ & 2012 \\
\hline $\begin{array}{l}\text { Perfil de usuários atendidos em uma unidade não } \\
\text { hospitalar de urgência(2). }\end{array}$ & Garcia VM; Reis RK. & $\begin{array}{l}\text { necessidade da implantação do acolhimento } \\
\text { entre profissionais de saúde. }\end{array}$ & 2014 \\
\hline $\begin{array}{l}\text { Do Prazer ao Sofrimento no Trabalho da } \\
\text { Enfermagem: O Discurso dos Trabalhadores }{ }^{(15)} \text {. }\end{array}$ & Kessler IA; Rug FBS. & $\begin{array}{l}\text { Acompanhamento psicológico de auto-ajuda para } \\
\text { reforçar o psicológico para enfrentamento do trabalho. }\end{array}$ & 2012 \\
\hline $\begin{array}{l}\text { A questão da saúde mental na vigilância em saúde } \\
\text { do trabalhador }{ }^{(16)} \text {. }\end{array}$ & Leão LH.; Gomez CM. & $\begin{array}{l}\text { Promover ações paranegociar mudanças que } \\
\text { promovam satisfação no trabalho. }\end{array}$ & 2012 \\
\hline $\begin{array}{l}\text { Trabalho de enfermagem em unidade de terapia } \\
\text { intensiva e sua interface com a sistematização da } \\
\text { assistência }^{(6)} \text {. }\end{array}$ & Massaroli, R. et al & Constancia de análise no processo de trabalho. & 2015 \\
\hline $\begin{array}{l}\text { Dimensionamento de enfermagem em sala de } \\
\text { emergência de um hospital-escola }{ }^{(17)} \text {. }\end{array}$ & $\begin{array}{l}\text { Paixao T; Couto R. } \\
\text { et al. }\end{array}$ & $\begin{array}{l}\text { Quadro de funcionário de emergência sem condizer } \\
\text { com a necessidade de funcionários no setor. }\end{array}$ & 2015 \\
\hline $\begin{array}{l}\text { Qualidade de vida no trabalho: perspectivas e } \\
\text { desafios atuais }{ }^{(18)} \text {. }\end{array}$ & Sampaio JR. & $\begin{array}{l}\text { Percepção de mudança faz-se necessário e estudar } \\
\text { a qualidade de vida no ambiente de trabalho que } \\
\text { possibilita o conhecimento das variáveis que } \\
\text { influenciam tal comportamento dos trabalhadores. }\end{array}$ & 2013 \\
\hline
\end{tabular}

Fonte: dados da pesquisa. 
sas filas de pessoas nos serviços de saúde, equipamentos quebrados, a falta de leitos hospitalares para atender a demanda da população, a falta de recursos financeiros e também a escassez derecursos materiais e humanos. Para manter os serviços de saúde operando com eficiência, são diariamente mostrados e verificados por todos ${ }^{(9)}$.

$\mathrm{Na}$ investigação(II) em relação aos achados, destacouse que a sobrecarga de tarefas pode gerar erros. Os conflitos de funções levam à insatisfação no trabalho e relações inter pessoais, a desvalorização profissional pode levar à desmotivação e abandono do trabalho, ocasionando altas taxas de absenteísmo, também ao ambiente insalubre, o que gera, na maioria das vezes, sobrecarga de trabalho para uns e priorizando outros. Dessa forma, ocorre a não realização de determinadas tarefas, ocasionando prejuízo ao cliente referente à assistência de enfermagem. A tocante à sua assistência, a arte do cuidar, apesar de ser uma das mais belas é também muitas vezes a mais difícil, pois lidar com o sofrimento do próximo quase sempre desencadeia, no profissional de enfermagem sentimentos de compaixão, sofrimento, resignação, impotência, estresse e depressão, considerando o setor de emergência com alto grau de fatores estressantes.

A visão de Qualidade de Vida no Trabalho veicula implícita e explicitamente três concepções nucleares de pensar o mundo da produção e o papel de seus principais protagonistas: (a) uma compreensão de ser humano retificada; (b) uma concepção instrumentalista/reducionista (c) uma concepção do trabalho humano, de organização centrada no produtivismo exacerbado ${ }^{(15)}$.

Atualmente algumas instituições tem se preocupado com a saúde física e mental de seus profissionais e vêm adotando estratégias para contornar o problema, seja por meio de exercícios físicos durante a jornada de trabalho; a ginástica laboral; ou pela implementação de técnicas de relaxamento e atendimentos psicoterápicos para os pro- fissionais, porque as instituições se conscientizaram que se os profissionais da enfermagem estiverem bem e em harmonia com o corpo e mente produzirão mais, e terão menos gastos com afastamentos por problemas de saúde ${ }^{(18)}$.

Os autores ${ }^{(16-20)}$ corroboram no sentido de a liderança interferir no comportamento e afastamento dos trabalhadores, descrevem que quando existe clareza nos objetivos da gestão e participação dos trabalhadores, a percepção para o novo muda. Relatam que acontece a diminuição dos afastamentos por doenças do sistema osteomuscular nos profissionais de saúde, e demonstra nos estudos a falta de importância devida dos gestores para as taxa de absenteísmo.

\section{CONSIDERAÇÕES FINAIS}

Esta investigação considera que o estresse do profissional de enfermagem é resultante de baixa remuneração, fazendo com que o trabalhador tenha mais de um vínculo empregatício, sobrecarga de trabalho e a não valorização do trabalho.Para que haja controle dos fatores estressantes no setor de emergência para os profissionais de enfermagem, é necessário a valorização dos distintos saberes com ênfase nas experiências dos profissionais.

O Profissional Enfermeiro no setor de emergência percebe os fatores de estresse nos quais está envolvido, e é de grande valia o estímulo como solução para melhor qualidade de vida do profissional de enfermagem, quando a instituição na qual está o trabalhador vinculado dispor de plano de carreira, aumento salarialadequado para seu nível de estudo, liderança democrática e não punitiva, assim como um ambiente sustentável contribuindo para que este profissional tenhaqualidade de vida. $O$ preparo do Enfermeiro deve ser para atuar emunidades diversificadas, considerando a humanização desde os integradores até com seus próprios pacientes, procurando desta forma melhor qualidade de vida. 


\section{REFERÊNCIAS}

I. Monte PF, et al. Estresse dos profissionais enfermeiros que atuam na unidade de terapia intensiva. Acta paul. enferm. 20I 3; 26(5): 42 I-427.

2. Garcia VM; Reis RK.Perfil de usuários atendidos em uma unidade não hospitalar de urgência. Rev. bras. enferm. 2014; 67(2):26I-267.

3.Machado MH;Vieira ALS; Oliveira E. Construindo o perfil da enfermagem. Enfermagem em foco. 2012; 3(3).

4. Silva N, Tolfo SR. Trabalho Significativo e Felicidade Humana: explorando aproximações. 2012.

5.Limada Silva JL; DiasCA. Discussão sobre as causas da Síndrome de Burnout e suas implicações à saúde do profissional de enfermagem.Aquichán, Bogotá. 2012; 12(2):।44-I59.

6. Massaroli R; et al. Trabalho de enfermagem em unidade de terapia intensiva e sua interface com a sistematização da assistência. Esc. Anna Nery, 20I5; 19(2):252-258.

7. Souza MT, Silva MD, Carvalho R. Revisão integrativa: o que é e como fazer. Rev Einstein. 2010; 8(1):102-6.

8. Andrade MAC; Artmann E; Trindade ZA. Humanização da saúde em um serviço de emergência de um hospital público: comparação sobre representações sociais dos profissionais antes e após a capacitação. Ciênc. saúde coletiva. 20II; I6(I): III5-II24.

9. Bezerra FN; Silva, TM; Ramos VP. Estresse ocupacional dos enfermeiros de urgência e emergência: Revisão Integrativa da Literatura.Acta paul. enferm. 20|2; 25(2):I5I-I56.

10.Camponogara S; Santos TM; Seiffert MA; et al. Cuidado Humanizado em Terapia Intensiva: Uma Revisão Bibliográfica. Rev.de Enfermagem. 201 I; I(I):I24-I32.

I I. Linhares MBM. Estresse precoce no desenvolvimento: impactos na saúde e mecanismos de proteção. Estud. psicol. 2016; 33(4):587-599.

12. Frias L; Lopes N. Considerações sobre o conceito de dignidade humana. Rev. direito GV. 20I5; I I (2):649-670.

13. FerreiraCM. Qualidade de vida no trabalho (QVT): do assistencialismo à promoção efetiva. Laboreal.20 I5; I I (2): 28-35.

14. Oenning NS; Carvalho FM.; Lima VMC. Indicadores de absenteísmo e diagnósticos associados às licenças médicas de trabalhadores da área de serviços de uma indústria de petróleo. Rev. bras. saúde ocup.2012; 37(I25): I 50- I 58.

15. Kessler IA; Rug FBS. Do Prazer ao Sofrimento no Trabalho da Enfermagem: O Discurso dos Trabalhadores, 2012.

16. Leao LH; Gomez CM. A questão da saúde mental na vigilância em saúde do trabalhador. Ciênc. saúde coletiva. 2014; 19(I2):4649-4658.

17. Paixao T; Couto R. et al. Dimensionamento de enfermagem em sala de emergência de um hospital-escola. Rev. esc. enferm. USP. 2015; 49(3):48I-487.

18. Sampaio JR. Qualidade de vida no trabalho: perspectivas e desafios atuais. Rev. Psicol., Organ. Trab.20 I2; I2(I): I2I-136. 